\title{
Magnetic states in wide annular structures
}

\author{
M. Kläui ${ }^{\mathrm{a})}$ and U. Rüdiger \\ Fachbereich Physik, Universität Konstanz, Universitätsstrasse 10, 78457 Konstanz, Germany \\ C. A. F. Vaz and J. A. C. Bland \\ Cavendish Laboratory, University of Cambridge, Madingley Road, Cambridge CB3 OHE, United Kingdom \\ S. Cherifi, ${ }^{\text {b) }}$ A. Locatelli, and S. Heun ${ }^{\text {c) }}$ \\ Sincrotrone Trieste, 34012 Basovizza, Trieste, Italy
}

\section{A. Pavlovska and E. Bauer}

Department of Physics and Astronomy, Arizona State University, Tempe, Arizona 85287

L. J. Heyderman

Laboratory for Micro- and Nanotechnology, Paul Scherrer Institut, CH-5232 Villigen PSI, Switzerland

(Presented on 1 November 2005; published online 24 April 2006)

The results of nonintrusive high-resolution imaging of the magnetic states present in wide Co rings with a small inner hole (1.7 $\mu \mathrm{m}$ outer diameter, $300 \mathrm{~nm}$ inner diameter, and $700 \mathrm{~nm}$ width) are presented. Rather than the reproducible onion state commonly found in rings, a prevalent triangle state is observed, with other magnetic states characteristic of disks also occurring. The uniformity and the magnetic states in these wide rings are found to be more typical of disks than of rings. Analyzing the energetics of the formation of the magnetic states, this is attributed to the reduced shape anisotropy in wide rings as compared to narrow rings. () 2006 American Institute of Physics. [DOI: $10.1063 / 1.2169485$ ]

The evolution of devices to smaller and smaller scales has fueled an increasing research interest in mesoscopic magnetic elements. In addition to insights into the underlying physical principles, mesoscopic structures have shown a potential for a range of applications in nanotechnology. These applications include memory elements in high-density storage media or miniaturized sensor elements. ${ }^{1,2}$ Intensive research has been devoted to understanding and, in particular, controlling the magnetic properties of small ferromagnetic elements. A key issue is the magnetic switching, since this determines whether a magnetic nanostructure can be utilized in applications. Narrow magnetic rings have shown to be a useful geometry, since particularly well-defined states are observed and the switching between these states has been found to be reproducible, simple, and fast. ${ }^{3-12}$ In most of the ring geometries investigated so far, micromagnetic simulations and magnetization measurements show the existence of two magnetic states: $:^{3,4,10,12}$ the flux-closure vortex state and the "onion" state, accessible reversibly from saturation and characterized by the presence of two opposite head-to-head walls. In narrow magnetic rings the magnetic states and the switching have been thoroughly studied, ${ }^{3-6,12,13}$ with particular emphasis on the head-to-head domain walls present in the onion state. ${ }^{12,14}$ In disks and similar topologically simply connected elements (ellipses, rectangles, etc.), in addition to the vortex state, more complex states $(s$ state, $c$ state, and triangle state) have been predicted and observed. ${ }^{15-20} \mathrm{~A}$ con-

\footnotetext{
${ }^{a)}$ Electronic mail: mathias.klaeui@uni-konstanz.de

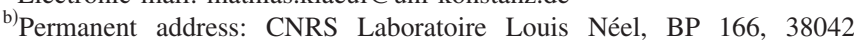
Grenoble, France.

${ }^{c}$ Present address: TASC-INFM Laboratory, Area Science Park, 34012 Basovizza, Trieste, Italy
}

sequence of the existence of different magnetic states with similar energies in disks is that after subjecting an array of nominally identical disks to the same field history, disks are found in different magnetic states. ${ }^{20}$ This is due to small edge irregularities or film defects, which cause energy barriers between the metastable states and the ground state. In narrow rings with a limited number of magnetic states, good reproducibility and uniformity of the magnetic states across an array have been observed. ${ }^{21}$

The switching in rings has been probed for a wide range of parameters, ${ }^{5,10,12,13}$ and in addition to the usually observed double switching between the onion and the vortex state, wide and thick rings exhibit a triple switching. This involves the "vortexcore" state, where one complete vortex core is present, and this state was found to be only stable under an applied field. ${ }^{12}$ In contrast to the switching processes, which have been studied for all ring widths ranging from very narrow rings with a ratio of inner to outer diameter of 1/1.3 to very wide rings with a ratio of $1 / 6,{ }^{13}$ the magnetic states have so far only been directly determined for narrow rings (ratios between $1 / 1.3$ and 1/3) and for disks. ${ }^{5,8,10,12,20,21}$ In disks there is no direct equivalence for the onion state in rings, but rather a number of possible states ( $c$ state, $s$ state, etc.). Thence a key question, which so far has not been addressed, is whether these states are confined to disks or whether they are also present in sufficiently wide rings. In this paper we determine by direct imaging the magnetic states in rings with widths of up to $700 \mathrm{~nm}$, corresponding to a ratio between the inner and outer diameter of 1/5.7.

We employ electron-beam lithography to define rings with an outer diameter $D=1.7 \mu \mathrm{m}$ and widths between 100 and $700 \mathrm{~nm}$. Since the edge definition is given by the 
electron-beam accuracy, the relative edge roughness is smaller in wide rings than in narrow rings. The edge definition of $>90 \%$ of the structures is better than $30 \mathrm{~nm}$. The maximum size deviation is $<10 \%$. For pattern transfer, a lift-off process is used. Polycrystalline cobalt films of $10 \mathrm{~nm}$ in thickness with a 2-nm-thick Au capping layer to prevent oxidation of the magnetic film were deposited on the patterned resist on silicon by molecular-beam epitaxy in an ultrahigh-vacuum deposition chamber (base pressure of 3 $\times 10^{-10}$ mbar. $^{22,23}$ Prior to imaging the magnetic states, we saturate the rings with a strong external field $(>5 \mathrm{kOe})$ and remove the Au layer by Ar-ion bombardment. Edge-to-edge spacings of $250 \mathrm{~nm}-1.5 \mu \mathrm{m}$ were used. Scanning electron microscopy images of rings fabricated with this technique are shown in Ref. 22.

To determine the spin structure of the magnetization configuration, the ring arrays were directly imaged with nonintrusive $\mathrm{x}$-ray magnetic circular dichroism photoemission electron microscopy (XMCDPEEM) at room temperature. In XMCDPEEM, the yield of secondary electrons created by excitation with circularly polarized light depends on the dot product $\mathbf{P} \cdot \mathbf{M}$ of the polarization $\mathbf{P}$ and the magnetization direction $\mathbf{M}$. The secondary electrons are used for imaging by a photoemission electron microscope (PEEM) and the yield difference is visible as magnetic contrast in the images. ${ }^{24}$ To understand the contribution of the different energy terms, micromagnetic simulations were computed using the OOMMF package. ${ }^{25}$ The parameters used are $M_{s}=1424$ $\times 10^{3} \mathrm{~A} / \mathrm{m}, A=3.3 \times 10^{-11} \mathrm{~J} / \mathrm{m}$, a damping constant of 0.01 , and a cell size of $5 \mathrm{~nm}$.

In Fig. 1(a) the typical onion state with transverse walls in a narrow ring, characterized by the triangular contrast, is visible. ${ }^{14}$ We now increase the width of the rings to $700 \mathrm{~nm}$ : part of an array of these rings, which have a small inner hole (inner diameter of $300 \mathrm{~nm}$ ), is presented in Fig. 1(b). Firstly, it is clear that the contrast is very different from the onion state in narrower rings, which means that the wide rings are in a different magnetic state, not previously observed in narrow rings. Secondly, we see that not all rings exhibit identical contrast, which means that different magnetic states are present in nominally identical rings, which have undergone the same magnetizing field history. Furthermore, after remagnetizing the sample, we sometimes observe that a given ring changes its magnetic state.

Let us first turn to the prevalent state observed, a magnified image of which is presented in Fig. 1(c). The spin structure of this state is completely different from the onion state, since it does not contain proper domain walls but rather a wavy spin structure. ${ }^{15,18,20}$ In Fig. 1(d) we present a micromagnetic simulation of such a ring. The simulation reproduces the experimentally observed spin structure very well. Since the magnetization in this state follows primarily the outer perimeter of the ring, there are only two positions indicated by the arrows in Fig. 1(d) where stray field enters or leaves the ring. While this magnetization configuration was not observed in narrow rings, theoretically this state has been predicted for disks by Zhu et al. ${ }^{15}$ and was observed experimentally in Co disks (triangle state) ${ }^{20}$ Due to the axial symmetry along the direction of the applied field, this state can
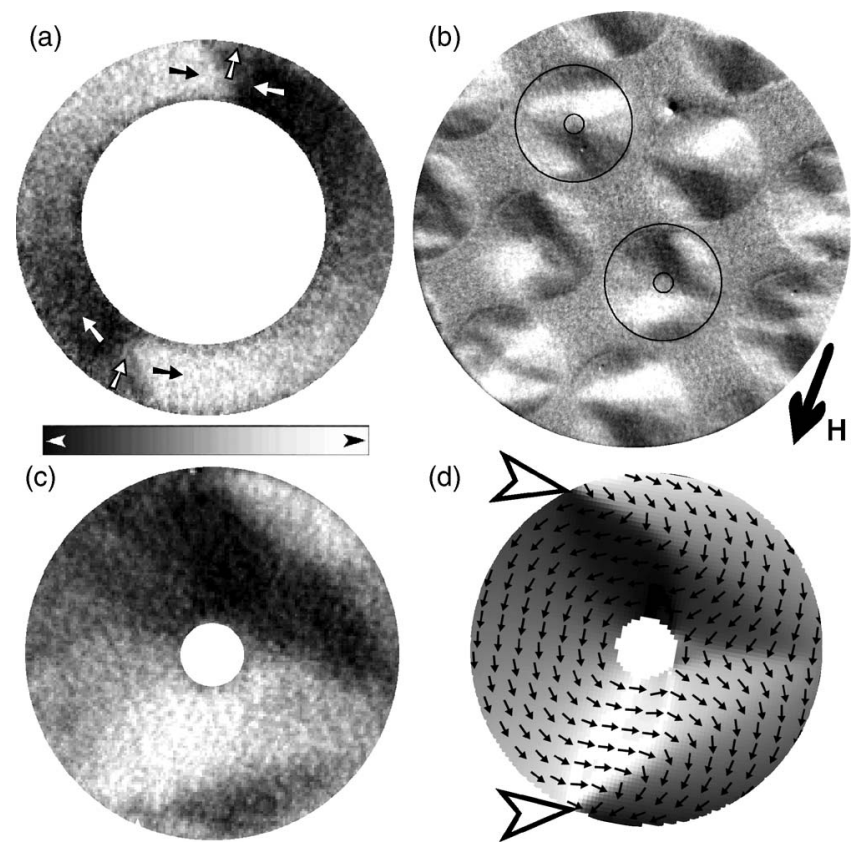

(d)

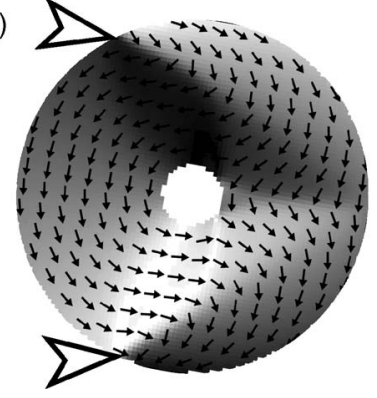

FIG. 1. (a) XMCDPEEM image of a 1.7- $\mu$ m-o.d., 350-nm-wide, 10-nmthick ring in the onion state with two transverse head-to-head domain walls and arrows indicating the magnetization directions. (b) An overview image (field of view of $6.5 \mu \mathrm{m}$ ) of 700 -nm-wide 10 -nm-thick rings with a $1.7 \mu \mathrm{m}$ outer diameter after a field was applied along the columns of rings the direction indicated by the arrow). The position of the two rings in the triangle state with an opposite sense of circulation is indicated. Most of the rings exhibit the triangle state; a high-resolution image is presented in (c). In (d), a micromagnetic simulation for such a ring is shown and it reproduces the experimentally observed spin structure very well. A black-white scale of the magnetic contrast is presented.

occur with two senses of circulation, as seen in Fig. 1(b), which are simply mirrored along the field direction and thus equivalent. For the narrow spacings shown in Fig. 1(b), the magnetostatic coupling leads to adjacent rings, in a row along the applied field direction, exhibiting triangle states with alternating senses of circulation. In contrast, in isolated rings we found the sense of circulation to be random. When calculating the energetics of this state, it becomes clear that the energy is much higher than the energy of the vortex state ${ }^{20}$ which is the ground state for this geometry. But when relaxing the field from saturation, the triangle state constitutes a local energy minimum, and to switch to the vortex state an energy barrier has to be overcome, so that we can observe the triangle state at zero field. Imaging other parts of the array, we find that some wide rings have already attained the vortex state [bottom left ring in Fig. 2(a)]. Apart from the triangle state and the vortex state, other more complicated states are found in the ring array [Figs. 2(a) and 2(b)]. The top right ring in Fig. 2(a) is in the $s$ state, which has previously been predicted and observed in disks and ellipses. ${ }^{15,18}$ Here the magnetization forms an $s$ shape following the perimeter of the ring and again creating two poles of magnetic charges [indicated by the arrows in Fig. 2(a)]. Compared to the triangle state, the magnetization is less bent than in the triangle state and the exchange energy is expected to be lower. ${ }^{20}$ While most of the rings have a perfectly round shape, some rings exhibit significant edge irregularities, as seen in Fig. 2(a). We have found that similarly to disks, these 


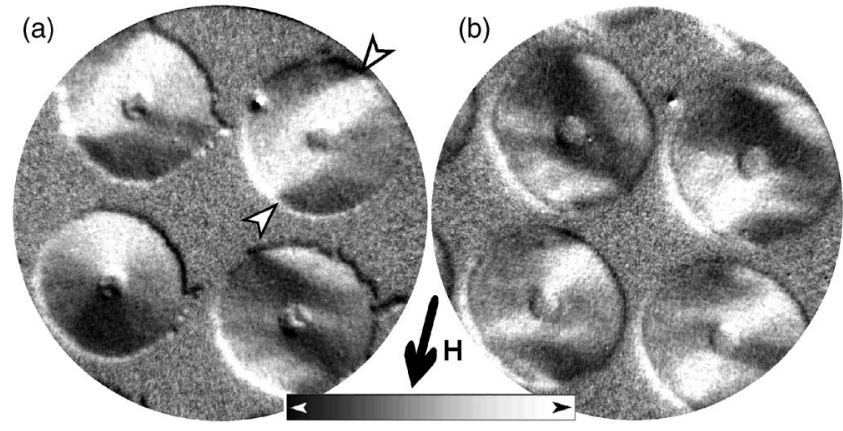

FIG. 2. Two overview images (field of view of $4.7 \mu \mathrm{m}$ ) of different parts of the array of 1.7- $\mu$ m-o.d., 700-nm-wide, 10-nm-thick rings. In addition to the triangle state, these rings exhibit a number of other different magnetic states. The bottom left ring in (a) exhibits the vortex state, while the top right ring the $s$ state. The black-white scale for the contrast is given as well as the field direction that was used for the saturation.

edge irregularities can stabilize even more complicated magnetization configurations, as seen in Fig. 2, and even rings with no apparent geometrical variations can exhibit complicated states. This is in stark contrast to narrow rings where we have only observed the onion state [e.g., Fig. 1(a)] after field application, even when similar edge irregularities are present.

All these observations show that the magnetic properties of wide rings, in particular, the magnetic states and the reproducibility and uniformity, are similar to disks rather than to narrow rings. So in ring structures with a small but significant inner diameter $(300 \mathrm{~nm})$, the inner hole is found not to play an important role for the magnetization configurations after relaxing a field. To comprehend the reason for this, we have to understand how the magnetic states are formed when the field is relaxed from saturation. Due to the shape anisotropy, the magnetization at the borders will align with the edges to minimize the stray field. In narrow rings the distance between the inner and outer edges is small and if the spins at the two edges are aligned, then the exchange will force the spins in between to align as well as to form the vortex or the onion state. If the ring is wide, on the other hand, then there are a large number of spins between the inner edge and the outer edge, which means that shape anisotropy is less important and thus the spins can twist much more easily to form more complicated domain structures. This is analogous to the reason why perfectly single-domain magnetization configurations are observed in narrow wires but more complicated domain structures in wide wires. ${ }^{16}$

The situation is different for the ground state in disks and rings of this geometry, which is the vortex state. In contrast to the states mentioned above, there is a direct equivalence for the vortex state in a disk, ${ }^{26}$ i.e., the vortex state in a ring. ${ }^{3,21}$ A fundamental difference between disks and rings is the existence of the central hole in a ring. This means that for the vortex state, there is a difference between the zero stray field of a ring without the vortex core and the stray field of a disk due to its out-of-plane vortex core. ${ }^{26,27}$ This fundamental difference holds for virtually any size hole as long as it is above the diameter of the vortex core (a few nanometers ${ }^{26}$ ), i.e., any ring is stray-field-free in the vortex state, regardless of the ring width, while a disk always has a stray field.

In conclusion we have investigated the magnetic states in wide ring structures. We have observed magnetic states, which are distinctly different from those commonly observed in narrow rings. In particular, the triangle state is found to prevail but other states, such as the $s$ state, are also observed. These states are reminiscent of the magnetic states observed in disks, which shows that, after relaxing a field from saturation, these wide rings behave like disks rather than like rings. Furthermore in contrast to narrow rings, the magnetic states are not only much more varied but also not as reproducible. This means that after remagnetization rings can be found in different magnetic states and that these states are much more susceptible to edge irregularities than for narrow rings. This difference can be understood from the energetics that govern the formation of the magnetization configuration and, in particular, the effect of the larger shape anisotropy for narrower rings that leads to better defined and more reproducible states.

This work was supported by the EU Research Infrastructure Action ("Integrating Activity on Synchrotron and Free Electron Laser Science"), the CMI Magnetoelectronic Devices project, the SPSRC Adventure Fund Supertoroids Project, the Deutsche Forschungsgemeinschaft (SFB 513), and the Landesstiftung Baden-Württemberg. One of the authors (E.B.) acknowledges support by the Office of Naval Research Grant No. N-000140210922, monitored by Chagaan Bataar, ONR, and by NATO. The XMCDPEEM imaging was carried out at ELETTRA.

${ }^{1}$ J.-G. Zhu et al., J. Appl. Phys. 87, 6668 (2000).

${ }^{2}$ M. M. Miller et al., Appl. Phys. Lett. 81, 2211 (2002).

${ }^{3}$ J. Rothman et al., Phys. Rev. Lett. 86, 1098 (2001).

${ }^{4}$ S. P. Li et al., Phys. Rev. Lett. 86, 1102 (2001).

${ }^{5}$ F. J. Castano et al., Phys. Rev. B 67, 184425 (2003).

${ }^{6}$ M. Kläui et al., Appl. Phys. Lett. 81, 108 (2002).

${ }^{7}$ S. Kasai et al., J. Magn. Magn. Mater. 239, 228 (2002).

${ }^{8}$ J. Bekaert et al., Appl. Phys. Lett. 81, 3413 (2002).

${ }^{9}$ X. Zhu et al., J. Appl. Phys. 93, 8540 (2003).

${ }^{10}$ T. Uhlig et al., Phys. Rev. Lett. 93, 047203 (2004).

${ }^{11}$ M. Kläui et al., Phys. Rev. Lett. 90, 097202 (2003).

${ }^{12}$ M. Kläui et al., Phys. Rev. B 68, 134426 (2003).

${ }^{13}$ M. Kläui et al., J. Magn. Magn. Mater. 290, 61 (2005).

${ }^{14}$ M. Kläui et al., Appl. Phys. Lett. 85, 5637 (2004).

${ }^{15}$ J.-G. Zhu et al., in Spin Dynamics in Confined Magnetic Structures I, Topics in Applied Physics, Vol. 83, edited by B. Hillebrands and K. Ounadjiela (Springer-Verlag, Berlin, 2002), p. 289.

${ }^{16}$ J. I. Martin et al., J. Magn. Magn. Mater. 256, 449 (2003).

${ }^{17}$ A. Fernandez et al., J. Appl. Phys. 87, 1395 (2000).

${ }^{18}$ X. Liu et al., J. Appl. Phys. 96, 5173 (2004).

${ }^{19}$ S. Cherifi et al., J. Appl. Phys. 98, 043901 (2005).

${ }^{20}$ C. A. F. Vaz et al., Phys. Rev. B 72, 224426 (2005).

${ }^{21}$ M. Kläui et al., J. Phys.: Condens. Matter 15, 985 (2003).

${ }^{22}$ M. Kläui et al., Appl. Phys. Lett. 86, 032504 (2005).

${ }^{23}$ L. J. Heyderman et al., J. Appl. Phys. 93, 10011 (2003).

${ }^{24}$ A. Locatelli et al., Surf. Rev. Lett. 9, 171 (2002).

${ }^{25}$ The public domain package is available at gams.nist.gov/oommf

${ }^{26}$ A. Wachowiak et al., Science 298, 577 (2002).

${ }^{27}$ C. A. F. Vaz et al., Phys. Rev. B 73, 054411 (2006). 